11

\title{
Генерация периодической последовательности мощных ультракоротких импульсов в цепочке связанных релятивистских ламп обратной и бегущей волн, работающих в режимах усиления и нелинейного компфнеровского подавления
}

\author{
(C) Н.С. Гинзбург, Э.Б. Абубакиров, М.Н. Вилков, И.В. Зотова, А.С. Сергеев \\ Институт прикладной фризики РАН, \\ 603950 Нижний Новгород, Россия \\ e-mail: ginzburg@appl.sci-nnov.ru
}

(Поступило в Редакцию 1 декабря 2017 г.)

Исследована динамика электронного СВЧ генератора, в цепь обратной связи которого включены лампы обратной и бегущей волны, работающие в режимах усиления и нелинейного компфнеровского поглощения соответственно. Показано, что излучение, генерируемое в такой системе, может представлять собой периодическую последовательность ультракоротких импульсов. Рассматриваемый механизм импульсной генерации аналогичен широко используемому в лазерной физике методу пассивной синхронизации мод.

DOI: $10.21883 /$ JTF.2018.08.46316.2581

\section{Введение}

В физике лазеров получил широкое применение механизм генерации ультракоротких импульсов (УКИ), основанный на эффекте пассивной синхронизации мод [1-3]. Указанный эффект реализуется при установке в резонатор лазера нелинейного элемента (амплитудного фильтра), поглощающего излучение малой амплитуды, и прозрачного для излучения большой интенсивности. В результате возникает синхронизация мод резонатора, при которой излучение лазера представляет собой периодическую последовательность ультракоротких импульсов высокой мощности.

Как показано в [4-7] указанный метод генерации УКИ может быть перенесен в СВЧ электронику. При этом генератор УКИ должен состоять из активного элемента (электронного усилителя) и нелинейного насыщающегося поглотителя, включенного в цепь обратной связи. Предлагаемая схема носит достаточно универсальный характер и может быть реализована как в широком частотном диапазоне, так и при различном уровне мощности генерируемых импульсов. Как известно, в сантиметровом диапазоне, а также в длинноволновой части миллиметрового диапазона субгигаваттные и гигаваттные уровни мощности излучения могут быть получены на основе релятивистских ламп обратной волны (ЛОВ), работающих как в режимах квазистационарной генерации [8-10], так и в короткоимпульсных режимах сверхизлучения (СИ) [11-14]. В последнем случае пиковая мощность электромагнитных импульсов может значительно превышать мощность относительно коротких запитывающих электронных пучков (сгустков). Такой эффект достигается за счет движения генерируемого электромагнитного импульса по электронному потоку и кумулятивного отбора энергии у различных фракций электронного потока. При этом электронный сгусток генерирует одиночный импульс высокой интенсивности, если его длина ограничена кооперативной длиной (длиной корреляции) [15]. Частота повторения импульсов СИ достигается за счет работы инжектора релятивистских электронных сгустков в импульсно-периодическом режиме и соответственно не превышает нескольких килогерц $[13,14]$. При этом генерируемые электромагнитные импульсы имеют некоррелированные фазы.

Как показано в настоящей работе, если взять в качестве активного элемента релятивистскую ЛОВ и замкнуть вход и выход цепью внешней обратной связи, в которую поместить нелинейный поглотитель (фильтр), то при квазистационарной инжекции электронного пучка можно обеспечить режим генерации периодической последовательности коротких импульсов. Интенсивность и длительность таких импульсов будет близка к импульсам СИ, но они будут иметь коррелированные фазы и высокую частоту повторения в сотни мегагерц, определяемую временем прохода возмущений по цепи обратной связи. При этом в качестве поглощающего

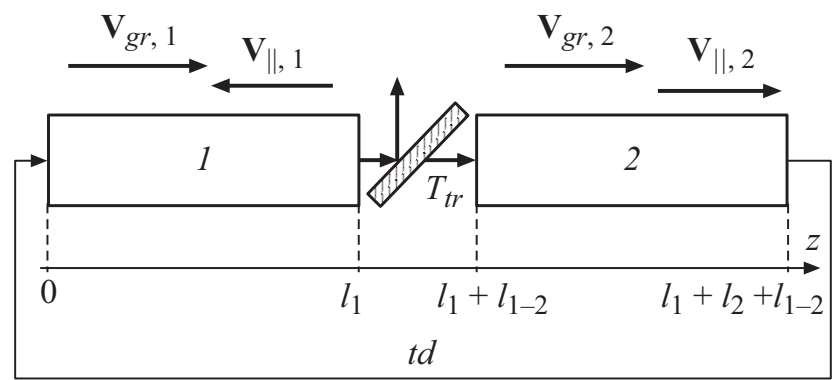

Рис. 1. Схема генератора ультракоротких импульсов, включающая ЛОВ в качестве активного элемента (1), и ЛБВ в качестве просветляющегося поглотителя (2), а также линию задержки и частично-прозрачное зеркало, выводящее излучение в полезную нагрузку. 
элемента можно использовать релятивистскую лампу бегущей волны (ЛБВ) (рис. 1), которая запитывается дополнительным независимым электронным пучком и работает в режиме компфнеровского подавления $[7,16]$.

\section{1. Модель и основные уравнения}

Рассмотрим схему двухсекционного генератора ультракоротких импульсов, состоящую из активного элемента на основе релятивистской ЛОВ (секция 1) и нелинейного поглотителя (секция 2) на основе релятивистской ЛБВ, работающей в режиме компфнеровского подавления. Кроме того, система включает полупрозрачное зеркало, которое возвращает часть излучения на вход ЛОВ по цепи обратной связи с определенной временной задержкой $t_{d}$ (рис. 1$)$.

В лампе обратной волны взаимодействие электронов со встречной волной

$$
E_{z}=\operatorname{Re}\left(A_{1}(z, t) E_{1}\left(\mathbf{r}_{\perp}\right) e^{i \omega t-i h_{1} z+i \bar{h}_{1} z}\right)
$$

происходит в условиях синхронизма

$$
\omega+h_{1}(\omega) V_{\|, 1} \approx \bar{h}_{1} V_{\|, 1} .
$$

В лампе бегущей волны взаимодействие электронов с попутной волной

$$
E_{z}=\operatorname{Re}\left(A_{2}(z, t) E_{2}\left(\mathbf{r}_{\perp}\right) e^{i \omega t-i h_{2} z-i \bar{h}_{2} z}\right)
$$

происходит в условиях синхронизма

$$
\omega-h_{2}(\omega) V_{\|, 2} \approx \bar{h}_{2} V_{\|, 2}
$$

Здесь $\omega$ - несущая частота, в качестве которой выберем частоту точного синхронизма в ЛОВ, $A_{s}(z, t)-$ медленно меняющиеся во времени $t$ и по продольной координате $z$ амплитуды волн в первой $(s=1)$ и второй $(s=2)$ секциях соответственно, $E_{s}(x, y)$ - поперечная структура электрического поля пространственных гармоник, $V_{\|, s}$ - невозмущенная поступательная скорость частиц, $h_{s}$ - продольное волновое число фундаментальной гармоники, $\bar{h}=2 \pi / d_{s}, d_{s}-$ период гофрировки волноводов.

В случае ультрарелятивистских энергий пучков $(\gamma \gg 1$, где $\gamma$ - Лоренц-фактор) электронно-волновое взаимодействие описывается уравнениями [17]

$$
\begin{gathered}
\left(\frac{\partial}{\partial z}+\frac{1}{V_{g r, 1}} \frac{\partial}{\partial t}\right) A_{1}=-\frac{E_{1}}{N_{1}} \frac{I_{1}}{\pi} \int_{0}^{2 \pi} e^{-i \theta_{1}} d \theta_{0}, \\
\left(\frac{\partial}{\partial z}-\frac{1}{V_{\|, 1}} \frac{\partial}{\partial t}\right) \gamma_{1}=-\operatorname{Re}\left(\frac{e A_{1} E_{1}}{m c^{2}} e^{i \theta_{1}}\right), \\
\left(\frac{\partial}{\partial z}-\frac{1}{V_{\|, 1}} \frac{\partial}{\partial t}\right) \theta_{1}=-\frac{\omega}{c}\left(1+\frac{1}{2 \gamma_{1}^{2}}\right)+\left(\bar{h}_{1}-h\right)
\end{gathered}
$$

для секции усиления на основе релятивистской ЛОВ (секция 1) и

$$
\begin{gathered}
\left(\frac{\partial}{\partial z}+\frac{1}{V_{g r, 2}} \frac{\partial}{\partial t}\right) A_{2}=\frac{E_{2}}{N_{2}} \frac{I_{2}}{\pi} \int_{0}^{2 \pi} e^{-i \theta_{2}} d \theta_{0}, \\
\left(\frac{\partial}{\partial z}+\frac{1}{V_{\|, 2}} \frac{\partial}{\partial t}\right) \gamma_{2}=-\operatorname{Re}\left(\frac{e A_{2} E_{2}}{m c^{2}} e^{i \theta_{2}}\right), \\
\left(\frac{\partial}{\partial z}+\frac{1}{V_{\|, 2}} \frac{\partial}{\partial t}\right) \theta_{2}=\frac{\omega}{c}\left(1+\frac{1}{2 \gamma_{2}^{2}}\right)-\left(\bar{h}_{2}+h\right)
\end{gathered}
$$

для секции нелинейного поглощения (секция 2) на основе релятивистской ЛБВ. В уравнениях использованы следующие обозначения: $\theta_{1}=\omega t+\left(\bar{h}_{1}-h_{1}(\omega)\right) z$ и $\theta_{2}=\omega t-\left(h_{2}(\omega)+\bar{h}_{2}\right) z-$ фазы электронов относительно синхронной встречной и попутной волн соответственно, $N_{s}$ - нормы рабочих мод, $V_{g r, s}$ - групповые скорости волн, $I_{s}$ - токи пучков в соответствующих секциях. Для упрощения будем далее считать, что обе секции запитываются пучками с одинаковой энергией частиц $\gamma_{0,1}=\gamma_{0,2}=\gamma_{0}$ и соответственно одинаковой невозмущенной поступательной скоростью $V_{\|, 1}=V_{\|, 2}=V_{\|}$. При этом настройка режима взаимодействия осуществляется за счет подбора параметров замедляющей системы.

Для дальнейшего анализа удобно провести переход к новым независимым переменным

$$
\tau=\frac{\omega}{2 c \gamma_{0}^{2}}\left(t+\frac{z}{V_{\|}}\right)\left(\frac{1}{V_{g r, 1}}+\frac{1}{V_{\|}}\right)^{-1}, \quad Z=\frac{\omega}{2 c \gamma_{0}^{2}} z
$$

В этих переменных взаимодействие в секции ЛОВ после нормализации опишется уравнениями

$$
\begin{gathered}
\frac{\partial a_{1}}{\partial \tau}+\frac{\partial a_{1}}{\partial Z}=-\frac{G_{1}}{\pi} \int_{0}^{2 \pi} e^{-i \theta_{1}} d \theta_{0}, \\
\frac{\partial u_{1}}{\partial Z}=-\operatorname{Re}\left(a_{1} e^{i \theta_{1}}\right), \quad \frac{\partial \theta_{1}}{\partial Z}=-\frac{1}{u_{1}^{2}}, \\
\left.u_{1}\right|_{Z=L_{1}}=1,\left.\quad \theta_{1}\right|_{Z=L_{1}}=\theta_{0} \in[0 ; 2 \pi),
\end{gathered}
$$

где $u_{1}=\gamma_{1} / \gamma_{0}, a_{1}=2 e E_{1} \gamma_{0} A_{1} / m c \omega, G_{1}=4 e I_{b, 1}\left|E_{1}\right|^{2} \times$ $\times \gamma_{0}^{3} / N_{1} m \omega^{2}$.

В этих же переменных процесс электронно-волнового взаимодействия в релятивистской ЛБВ опишется уравнениями

$$
\begin{gathered}
\frac{\partial a_{2}}{\partial Z}+\frac{2 s}{\beta_{\|}} \frac{\partial a_{2}}{\partial \tau}+s\left(\frac{1}{\beta_{g r, 2}}-\frac{1}{\beta_{\|}}\right) \frac{\partial a_{2}}{\partial \tau}=\frac{G_{2}}{D} \frac{1}{\pi} \int_{0}^{2 \pi} e^{-i \theta_{2}} d \theta_{0}, \\
\frac{\partial u_{2}}{\partial Z}+\frac{2 s}{\beta_{\|}} \frac{\partial u_{2}}{\partial \tau}=-D \operatorname{Re}\left(a_{2} e^{i \theta_{2}}\right),
\end{gathered}
$$




$$
\begin{gathered}
\frac{\partial \theta_{2}}{\partial Z}+\frac{2 s}{\beta_{\|}} \frac{\partial \theta_{2}}{\partial \tau}=\left(\frac{1}{u_{2}^{2}}-\Delta_{2}\right), \\
\left.u_{2}\right|_{Z=L_{1}}=1,\left.\quad \theta_{2}\right|_{Z=L_{1}}=\theta_{0} \in[0 ; 2 \pi),
\end{gathered}
$$

где $u_{2}=\gamma_{2} / \gamma_{0}, \quad G_{2}=4 e I_{b, 2}\left|E_{2}\right|^{2} \gamma_{0}^{3} / N_{2} m \omega^{2}, \quad a_{2}=2 e \times$ $\times E_{1} \gamma_{0} A_{2} \sqrt{N_{2}} / m c \omega \sqrt{N_{1}}, \quad s=\left(1 / \beta_{g r, 1}+1 / \beta_{\|}\right)^{-1}, \quad \Delta_{2}=$ $=2 \gamma_{0}^{2}\left(c / V_{p h, 2}-1\right), \quad V_{p h, 2}=\omega /\left(\bar{h}_{2}+h_{2}\right), \quad D=E_{2} \times$ $\times \sqrt{N_{1}} / E_{1} \sqrt{N_{2}}$.

В дальнейшем будем считать, что поступательная скорость электронов в этой секции $V_{\|}=c \beta_{\|}$близка к групповой скорости волны $V_{g r, 2}=c \beta_{g r, 2}$ и обе эти скорости близки к скорости света. Соответственно отличием указанных скоростей при анализе работы ЛБВ в режиме нелинейного поглотителя можно пренебречь. Данное условие эквивалентно требованию минимизации инерционности поглотителя в физике лазеров, где с этой целью обычно используются ячейки Керра [3]. В этом случае участки огибающей с большой и малой амплитудой не оказывают взаимного влияния, что очевидно увеличивает контраст между ними при прохождении насыщающегося поглотителя.

Как следует из схемы генератора, представленной на рис. 1, амплитуды полей на выходе ЛОВ и на входе в ЛБВ, которая играет роль нелинейного поглотителя, связаны следующим граничным условием:

$$
\left.a_{2}\right|_{Z=L_{2}}=\left.T_{t r} a_{1}\right|_{Z=L_{1}},
$$

где $T_{t r}$ - комплексный коэффициент передачи. Остальная доля мощности выводится в полезную нагрузку. Задержкой сигнала при прохождении промежутка $l_{1-2}$ между указанными секциями для простоты пренебрегаем. После прохождения поглотителя сигнал через линию задержки с нормированным временем запаздывания $T_{d}=\left(t_{d} \omega / 2 c \gamma_{0}^{2}\right)\left(1 / V_{g r, 1}+1 / V_{\|}\right)^{-1}$ снова поступает на вход ЛОВ, чему соответствует граничное условие

$$
\left.a_{1}\right|_{Z=0}=\left.a_{2}\left(\tau-T_{d}\right)\right|_{Z=L_{1}+L_{2}} .
$$

В дальнейшем для описания характеристик УКИ генератора удобно ввести коэффициент конверсии [11], определяемый как отношение мощности излучения, выводимой в полезную нагрузку, к мощности электронного пучка, запитывающего усилительную секцию:

$$
K=\frac{P_{\text {peak }}}{P_{\text {beam }}}=\frac{\left.\left|a_{1}\right|^{2}\right|_{Z=L_{1}}}{4 G_{1}}\left(1-\left|T_{t r}\right|^{2}\right),
$$

а также электронный КПД для каждой из секций

$$
\eta_{s}=\frac{\hat{\eta}_{s}}{\left(1-\gamma_{0, s}^{-1}\right)}, \quad \hat{\eta}_{s}=\frac{1}{2 \pi} \int_{0}^{2 \pi}\left(1-u_{s}\right) d \theta_{0} .
$$

Спектр выходного сигнала определяется соотношением

$$
S_{\Omega}=\left.(1 / 2 \pi) \int_{-\infty}^{+\infty} a_{1}(\tau)\right|_{Z=L_{1}} e^{-i \Omega \tau} d \tau .
$$
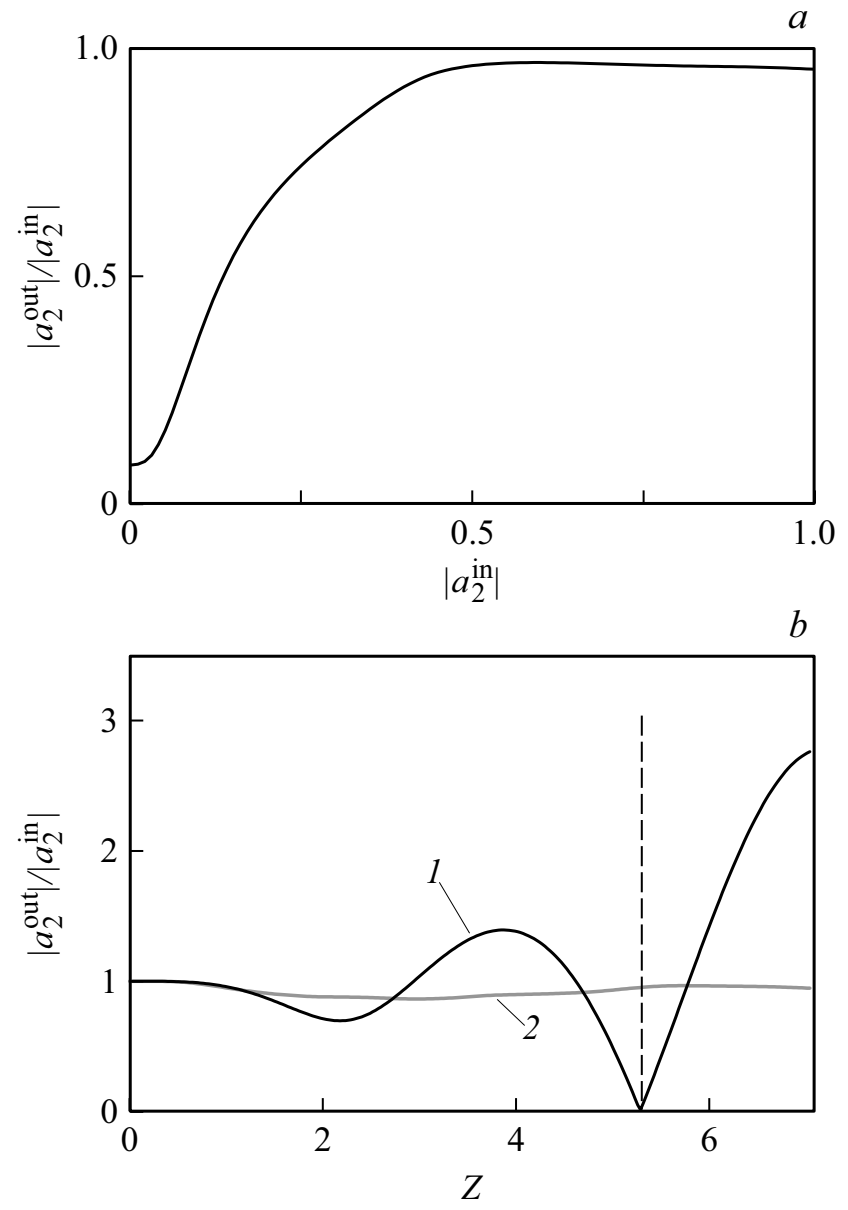

Рис. 2. Коэффициент прохождения $\left|a_{2}^{\text {out }}\right| /\left|a_{2}^{\text {in }}\right|$ компфнеровского поглотителя в зависимости: $a-$ от амплитуды входного сигнала $\left|a_{2}^{\text {in }}\right|$ при оптимальной длине секции поглощения $L_{2}=5.3$, $b-$ в зависимости от длины секции поглощения $L_{2}$ при двух значениях амплитуды, соответствующих слабому $\left(\left|a_{2}^{\text {in }}\right|=0.01\right.$, кривая 1$)$ и сильному $\left(\left|a_{2}^{\text {in }}\right|=1\right.$, кривая 2$)$ сигналам. $(G=0.06$, $\Delta=-0.32, D=1,2 s / \beta_{\|}=1$.)

\section{2. Результаты моделирования}

Продемонстрируем сначала, что релятивистская ЛБВ в области расстроек синхронизма, соответствующих режиму компфнеровского подавления (фазовая скорость синхронной компоненты поля превосходит поступательную скорость электронов), обладает свойствами насыщающегося поглотителя. На рис. 2, $a$ показана зависимость коэффициента прохождения сигнала $\left|a_{2}^{\text {out }}\right| /\left|a_{2}^{\text {in }}\right|$ от амплитуды падающего сигнала $\left|a_{2}^{\text {in }}\right|$ при оптимальной длине поглотителя $L_{2}=5.3$. Видно, что сигналы малой амплитуды практически полностью подавляются, в то время как сигналы большой амплитуды проходят без заметного поглощения. Представленные на рис. 2, $b$ зависимости коэффициента прохождения от продольной координаты для сигналов малой и большой интенсивности подтверждают сделанное выше заключение. 
Моделирование динамики полной системы, в цепь обратной связи которой включены ЛОВ в качестве активного усилительного элемента и ЛБВ в качестве нелинейного фильтра, показало возможность генерации в ней периодической последовательности ультракоротких импульсов (рис. 3), формирующихся в результате синхронизации большого числа продольных мод резонатора, образованного петлей обратной связи. В данном случае имеет место мягкий режим самовозбуждения,
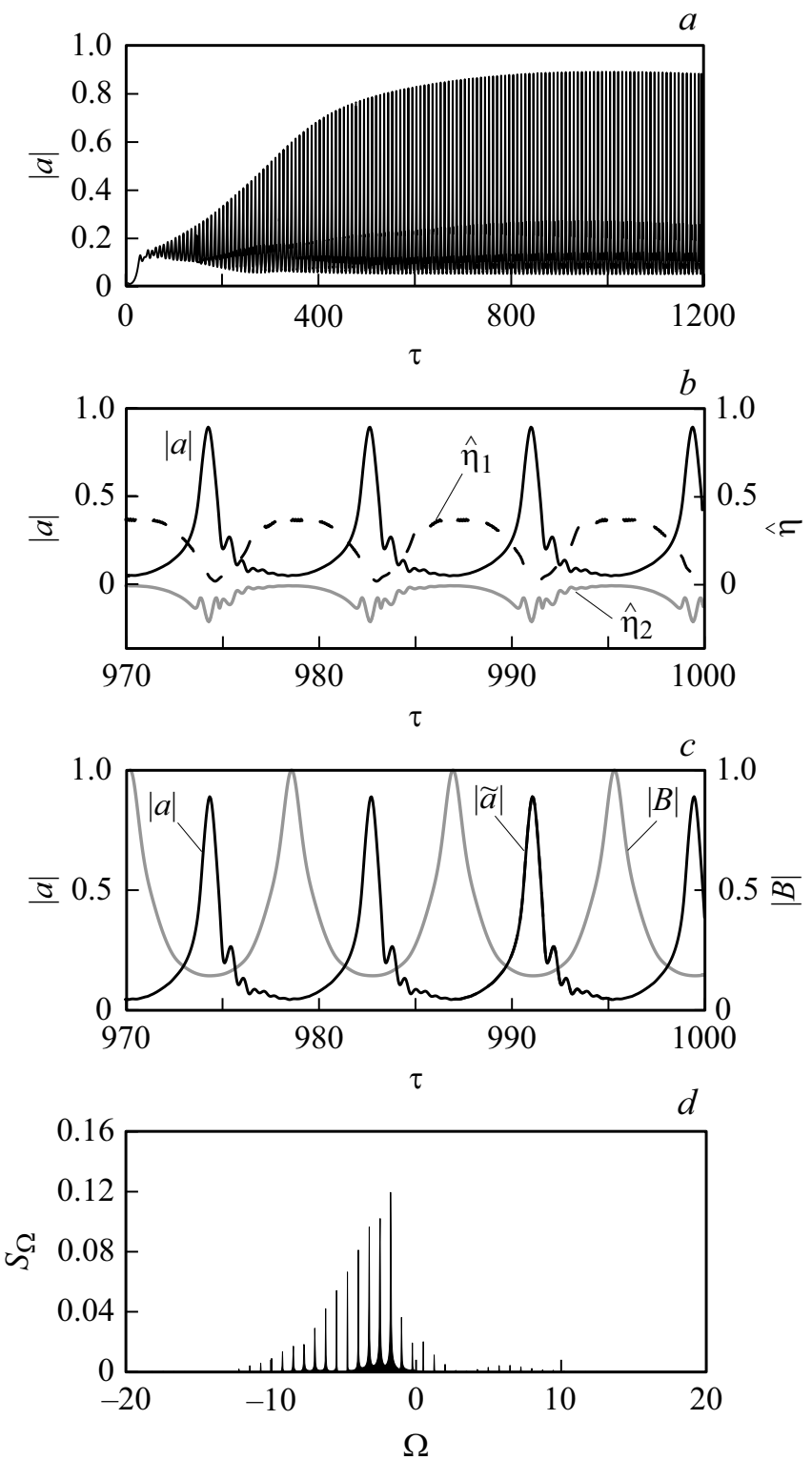

Pис. 3. Установление генерации УКИ в мягком режиме самовозбуждения: $a-$ полный сценарий переходного процесса, $b$ - профиль импульсов в увеличенном масштабе (КПД усилительной секции показан штрихом, а для секции поглощения - серым цветом), $c$ - модуль взаимной корреляционной функции $|B|$ и огибающая генерируемого излучения $|a|(|\tilde{a}|-$ тестовый импульс, с которым сравниваются остальные импульсы при вычислении корреляционной функции), $d-$ спектр излучения $\left(L_{1}=6, G_{1}=0.03, L_{2}=5.3, G_{2}=0.06, \Delta_{2}=-0.37\right.$, $\left.D=1,2 s / \beta_{\|}=1,\left|T_{t r}\right|=0.9, \arg T_{t r}=0, T_{d}=10.8\right)$.

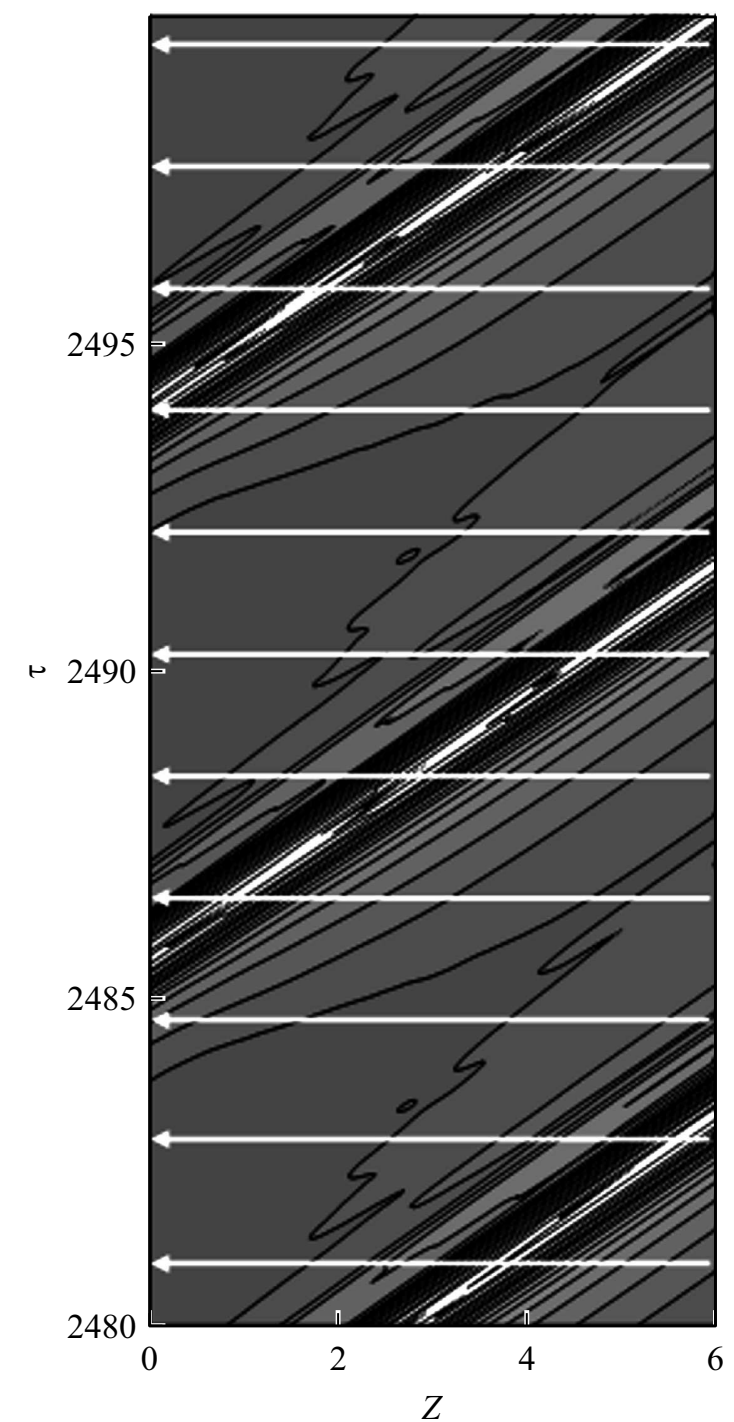

Рис. 4. Диаграмма, иллюстрирующая процесс смещения в переменных $Z$ и $\tau$, электромагнитного импульса относительно электронного пучка, приводящего к кумулятивному отбору энергии у различных фракций. Стрелками показаны электронные характеристики, направленные в отрицательном направлении оси $Z$.

когда генерация развивается из малых начальных шумов. При этом сначала возникает квазистационарная генерация, которая постепенно трансформируется в генерацию коротких импульсов. Как видно из рис. 4, формирующийся микроволновый импульс в секции ЛОВ последовательно (кумулятивно) отбирает энергию у различных электронных фракций. В результате, как и в режимах сверхизлучения [11-14], пиковая мощность УКИ импульсов оказывается существенно выше не только мощности излучения в стационарном режиме генерации, но и заметно превосходит мощность электронного пучка в усиливающем элементе. При выбранных параметрах коэффициент конверсии $K$, определяемый соотношением (12), достигает значения 1.2. При этом, как следует 

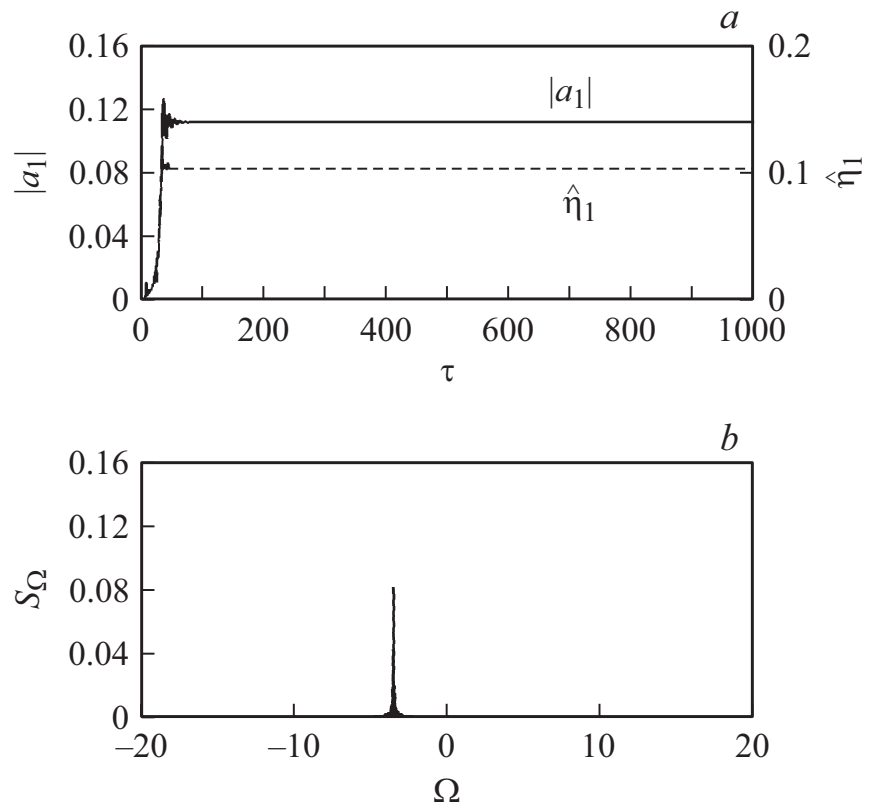

Рис. 5. Лампа обратной волны в режиме свободной генерации в отсутствие обратной связи: $a-$ временные зависимости амплитуды генерируемого сигнала и КПД; $b-$ спектр излучения $\left(T_{t r}=0\right.$, остальные параметры совпадают с приведенными в подписи к рис. 3.)

из рис. $3, b$, электронный КПД ЛОВ является в среднем по времени положительной величиной, т.е. электронный пучок этой секции отдает энергию излучению. В то же время КПД пучка, запитывающего вторую секцию генератора УКИ, отрицателен, т.е. электроны этого пучка в процессе нелинейного подавления частично забирают энергию поступающего в указанную секцию излучения.

Отметим, что генерируемые импульсы имеют высокую степень фазовой корреляции (рис. 3,c), что подтверждается при вычислении взаимной корреляционной функции между тестовым импульсом излучения $\tilde{a}(\tau)$ и полной последовательностью импульсов $a(\tau)$

$$
B(\tau)=\frac{\frac{1}{T} \int_{0}^{T} \tilde{a}\left(\tau^{\prime}\right) a^{*}\left(\tau^{\prime}-\tau\right) d \tau^{\prime}}{\left(\frac{1}{T} \int_{0}^{T}\left|\tilde{a}\left(\tau^{\prime}\right)\right|^{2} d \tau^{\prime} \cdot \frac{1}{T} \int_{\tau}^{T+\tau}\left|a\left(\tau^{\prime}\right)\right|^{2} d \tau^{\prime}\right)^{1 / 2}},
$$

где интервал $T$ представляет собой период следования импульсов.

Важно подчеркнуть, что релятивистская ЛОВ с параметрами, использованными в приведенном выше моделировании процесса генерации УКИ, при размыкании цепи обратной связи работает в стационарном режиме с выходной амплитудой примерно в 5 раз меньшей пикового значения в импульсном режиме (рис. 5). При этом если эту же ЛОВ поместить в цепь обратной связи с тем же коэффициентом передачи $\left|T_{t r}\right|=0.9$, то реализуется автомодуляционный квазипериодический режим генерации (рис. 6). Импульсы, генерируемые в этом режиме, сопоставимы по амплитуде с импульсами, генерируемыми при наличии насыщающегося поглотителя. Однако как форма импульсов, так и период их следования варьируют во времени. Кроме того, длительность указанных импульсов заметно превосходит длительность импульсов в режиме синхронизации мод.

Следует также отметить, что для исследуемой схемы существует зависимость режима генерации от фазового набега сигнала в цепи обратной связи. Так, на рис. 7 представлена последовательная смена режима генерации при изменении фазы коэффициента передачи $T_{t r}$. Видно, что при фазе $11 \pi / 9$ режим короткоимульсной генерации переходит в режим генерации монохроматического излучения, но далее при стремлении фазы к $2 \pi$ в системе снова реализуется импульсная генерация. Аналогичная смена режимов возникает при изменении
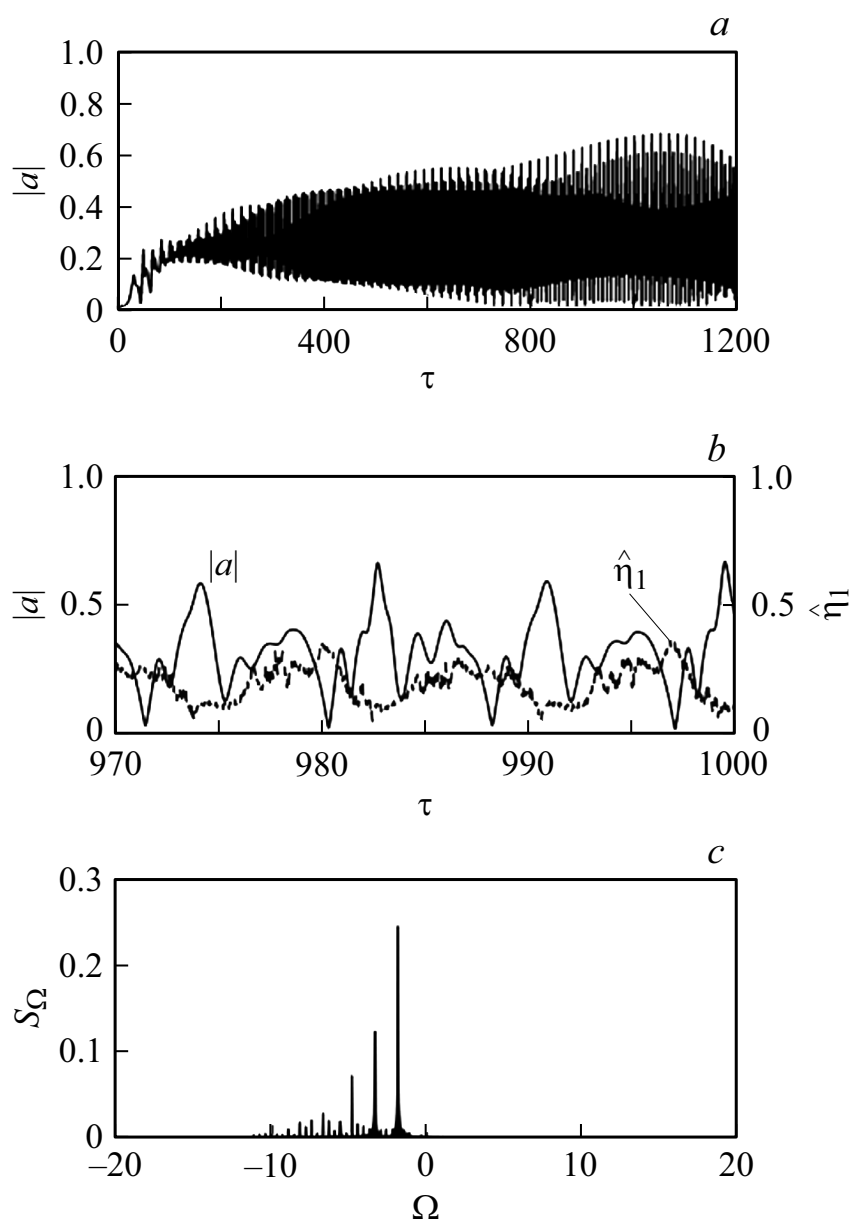

Рис. 6. Режим хаотической автомодуляции в присутствии обратной связи, но с выключенной поглощающей секцией: $a-$ временная зависимость амплитуды поля на выходе усилителя; $b$ - то же и текущий электронный КПД (серая линия) в развернутом по времени масштабе, $c-$ спектр излучения $\left(G_{2}=0\right.$, остальные параметры совпадают с приведенными в подписи к рис. 3 ). 

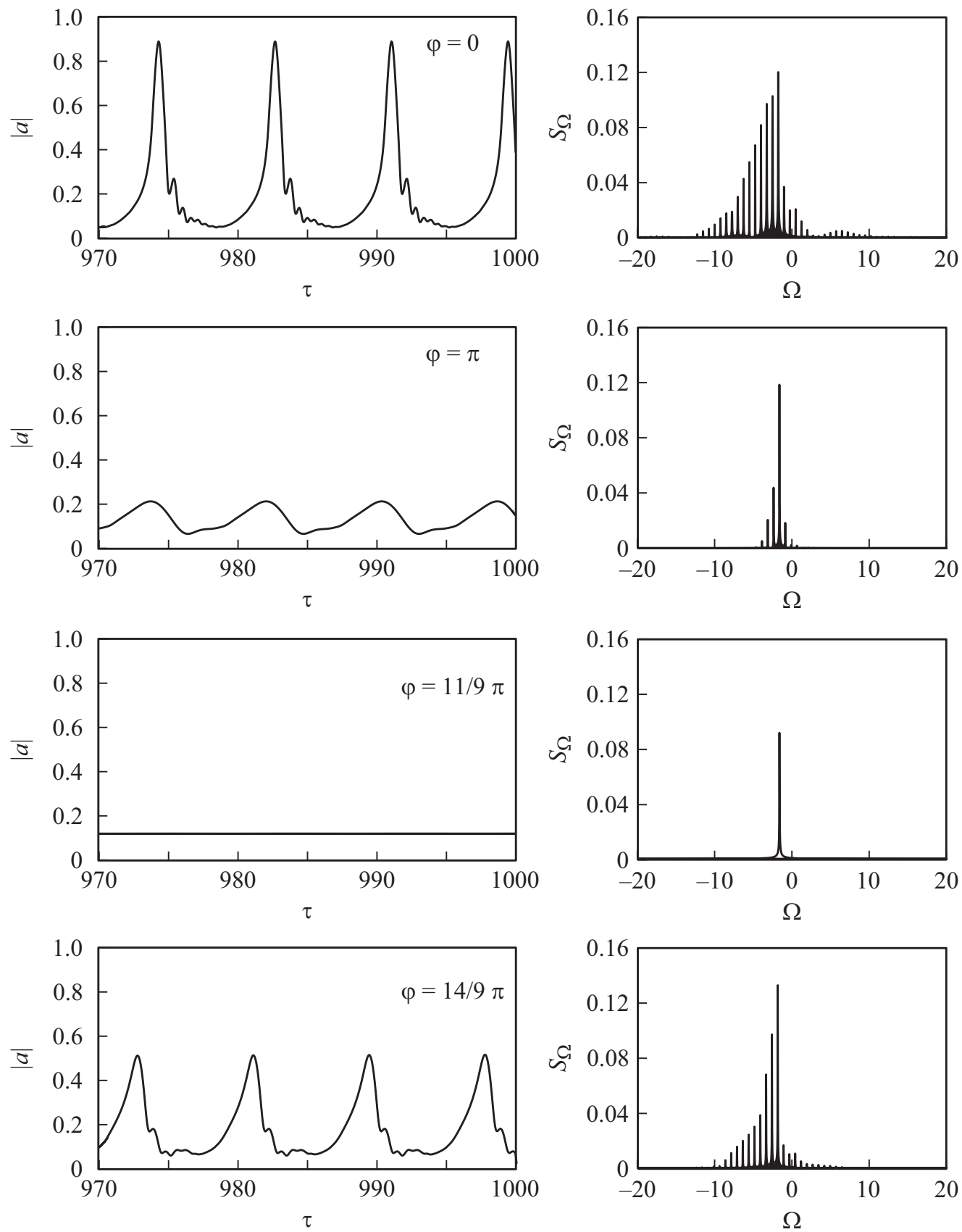

Рис. 7. Последовательная смена режима генерации при изменении фазы коэффициента передачи $\varphi=\arg T_{t r}$, левый столбец временные зависимости амплитуды генерируемого сигнала, правый столбец - спектр сигнала (параметры совпадают с приведенными в подписи к рис. 3).

времени задержки сигнала в цепи обратной связи. При этом режим генерации в отсутствие нелинейного поглотителя практически не меняется, т. е. сохраняется режим хаотической автомодуляции.

\section{Заключение}

Проведем в заключение оценку параметров генерируемых УКИ импульсов для случая, когда в качестве активного элемента используется релятивистская ЛОВ 3-х cm диапазона с рабочей волной $\mathrm{TM}_{01}$. Предположим, что средний радиус волновода $1.25 \mathrm{~cm}$, период гофрировки $1.7 \mathrm{~cm}$ и ее амплитуда $0.07 \mathrm{~cm}$, длина пространства взаимодействия $20 \mathrm{~cm}$. ЛОВ запитывается трубчатым электронным пучком с радиусом $0.9 \mathrm{~cm}$, энергией частиц $500 \mathrm{keV}$ и током $4 \mathrm{kA}$. Указанным физическим параметрам соответствуют следующие нормированные величины $L_{1}=6$ и $G_{1}=0.03$. В этом случае при оптимальных параметрах секции поглощения коэффициент конверсии, согласно рис. $3, a$, составит $K=1.2$, а пиковая мощность 
генерируемых импульсов может достигать $2.4 \mathrm{GW}$ при длительности по полувысоте - 450 ps. Частота следования импульсов при времени задержки $5 \mathrm{~ns}$ составляет $260 \mathrm{MHz}$.

Работа выполнена при поддержке Российского научного фонда, грант № 16-42-01078.

\section{Список литературы}

[1] Haus H.A. // IEEE J. Sel. Top. Quant. 2000. Vol. 6. P. 11731185.

[2] Крюков П.Г. // Квантовая электроника. 2001. Т. 31. № 2. C. 95-119.

[3] Brabec T., Spielmann Ch., Curley P.F., Krausz F. // Opt. Lett. 1992. Vol. 17. N 18. P. 1292-1294.

[4] Гинзбург Н.С., Денисов Г.Г., Вилков М.Н. и др. // Письма в ЖТФ. 2015. Т. 41. Вып. 17. С. 44-52.

[5] Ginzburg N.S., Denisov G.G., Vilkov M.N. et al. // Phys. Plasmas. 2016. Vol. 23. P. 050702-1-050702-4.

[6] Ginzburg N.S., Denisov G.G., Vilkov M.N. et al. // Phys. Plasmas. 2017. Vol. 24. P. 023103-1-023103-5.

[7] Гинзбург Н.С., Абубакиров Э.Б., Вилков М.Н. и др. // Письма в ЖТФ. 2015. Т. 43. Вып. 18. С. 47-55.

[8] Ковалёв Н.Ф., Петелин М.И., Райзер М.Д. и др. // Письма в ЖЭТФ. 1973. Т. 18. Вып. 4. С. 232.

[9] Carmel Y., Ivers J., Kribel R. E., Nation J. // Phys. Rev. Lett. 1974. Vol. 33. N 21. P. 1278-1282.

[10] Абубакиров Э.Б., Белоусов В.И., Варганов В.Н. и др. // Письма в ЖТФ. 1983. Т. 9. Вып. 9. С. 533-536.

[11] Ельчанинов А.А., Коровин С.Д., Ростов В.В. и др. // Письма в ЖЭТФ. 2003. Т. 77. Вып. 6. С. 314-318.

[12] Коровин С.Д., Любутин С.К., Месяи Г.А. и др. // Письма в ЖТФ. 2004. Т. 30. Вып. 17. С. 23-32.

[13] Коровин С.Д., Месяи, Г.А., Ростов В.В. и др. // Письма в ЖТФ. 2004. Т. 30. Вып. 3. С. 68-74.

[14] Korovin S.D., Eltchaninov A.A., Rostov V.V. et. al. // Phys. Rev. E. 2006. Vol 74. P. 016501.

[15] Гинзбург Н.С., Новожсилова Ю.В., Сергеев А.С. // Письма в ЖТФ. 1996. Т. 55. Вып. 9. С. 39-44.

[16] Kompfner R. // J. Brit. IRE. 1950. Vol. 10. N 8-9. P. 283-289.

[17] Гинзбург Н.С., Кузнецов С.П., Федосеева Т.Н. // Изв. вузов. Радиофизика. 1978. Т. 21. Вып. 7. С. 1037-1052. 